\title{
GERAÇÃO AUTOMÁTICA DE MODELO CRUSTAL UTILIZANDO PRIMEIRAS CHEGADAS DE LEVANTAMENTO DE REFRAÇÃO SÍSMICA PROFUNDA
}

Frederico Xavier de Melo, Departamento de Engenharia Elétrica, Universidade de Brasília, UnB Geovany Araújo Borges, Departamento de Engenharia Elétrica, Universidade de Brasília, UnB José Eduardo Pereira Soares, Instituto de Geociências, Universidade de Brasília, UnB

Copyright 2004, SBGf - Sociedade Brasileira de Geofísica

Este texto foi preparado para a apresentação no I Simpósio de Geofísica da Sociedade Brasileira de Geofísica, São Paulo, 26-28 de setembro de 2004. Seu conteúdo foi revisado pela Comissão Tecno-científica do I SR-SBGf mas não necessariamente representa a opinião da SBGf ou de seus associados. E proibida a reprodução total ou parcial deste material para propósitos comerciais sem prévia autorização da SBG.

\section{Resumo}

Este trabalho aborda o problema da classificação dos alinhamentos da fase direta $(\mathrm{Pg})$ e refratada da Moho (Pn), a partir do tempo de trânsito das ondas elásticas em estudos de refração sísmica profunda. O método empregado para classificação baseia-se na técnica de divisão e fusão em agrupamento difuso por protótipo. A validação do método proposto é feita a partir de uma base composta por dados reais e sintéticos. Este trabalho é parte de um algoritmo que propõe a geração automática de um modelo 1D-médio da crosta continental a partir dos traços adquiridos a partir de fontes controladas.

\section{Introdução}

A profundidade e topografia da descontinuidade de Moho são informações necessárias para estudo da litosfera continental e estruturação tectônica regional. A Moho é normalmente mapeada por métodos sísmicos, utilizando fontes sísmicas naturais (terremotos) e/ou controladas (explosões). Dentre os métodos sísmicos com fonte controlada destaca-se a refração profunda. Amplamente utilizada nos países do hemisfério norte e Austrália (Christensen et al., 1995), a refração profunda é ainda embrionária no Brasil. Destacam-se os trabalhos realizados na Província Tocantins (Perosi, 2000, Berrocal et al., 2004; Soares, 2005, Melo, 2005) e aqueles propostos para a Província Borborema (Fuck et al., 2005).

Este trabalho é parte de pesquisa que propõe algoritmo para geração de modelo 1D-médio da crosta continental a partir da leitura e interpretação automática do tempo de trânsito das primeiras chegadas e da reflexão da Moho de levantamentos de refração sísmica profunda. O algoritmo se divide: i) leitura automática das primeiras chegadas; ii) identificação dos alinhamentos das fases diretas $(\mathrm{Pg})$ e refratada na Moho $(\mathrm{Pn})$ e determinação de modelo da crosta com espessura mínima; iii) identificação das reflexões da Moho $(\mathrm{PmP})$ e cálculo do modelo 1D-médio. A primeira etapa foi testada com sucesso em telessísmos (Melo et al., 2005). Em dados de refração os resultados estão sendo apresentados e discutidos nesse encontro. A segunda etapa é o que discute este resumo. A terceira etapa é o grande desafio, pois envolve a determinação de fases internas relativas às chegadas da ondas refletidas na Moho.

As primeiras chegadas de levantamento de refração sísmica profunda correspondem às fases das ondas direta e refratada na descontinuidade de Moho. Para meios homogêneos e isotrópicos essas fases se alinham segundo segmentos de retas que, uma vez determinados, permitem o cálculo do modelo crustal com espessura mínima.

A observação do tempo de trânsito das ondas sísmicas exerce papel crucial nos estudos sobre a estrutura crustal da Terra. Aliada à teoria de propagação das ondas elásticas, possíveis soluções para o problema inverso, ou seja, inferir sobre as propriedades físicas do meio a partir da interpretação dos dados adquiridos, podem ser obtidas.

A curva de tempo de trânsito utlizada no estudos sobre a crosta e o manto superior é composta pelas leituras dos tempos de chegada das fases, a partir de uma fonte controlada com localização conhecida, em sensores distribuídos ao longo de uma linha sísmica. Dentre os métodos utilizados para obter dados provenientes da curva de tempo de trânsito, a refração sísmica permite extrair informações sobre a velocidade e a profundidade de diversas camadas a partir da observação das fases que sofrem alterações críticas na interface entre duas camadas. Sua vasta escalabilidade de aplicação permite - estudo desde camadas rasas, com profundidade inferior aos 100 metros, até investigações sobre a organização da estrutura crustal e do manto superior da Terra.

O problema abordado neste trabalho (item ii) resume-se na identificação do grupo de leituras correspondentes a onda direta $(\mathrm{Pg})$ e a onda refratada na Moho $(\mathrm{Pn})$, e a aproximação dessas leituras para segmentos de retas correspondentes aos alinhamentos das ondas $\mathrm{Pg}$ e $\mathrm{Pn}$. O algoritmo proposto utiliza método matemático de classificação (Split-Merge and Fuzzy) para determinar o grupo de chegadas correspondentes às ondas direta e refratada e, empregando técnicas de regressão linear por mínimos quadrados ponderados, determinar a equação dos alinhamentos das fases, de maneira a calcular um modelo crustal inicial com espessura mínima. 


\section{Metodologia}

A curva tempo-distância correspondente as ondas Pg e $\mathrm{Pn}$, primeiras quebras em $\mathrm{N}$ estações distribuídas ao longo de uma linha de refração sísmica, pode ser representada como $Z=\left\{\left(x_{j}, t_{j}\right) \mid j=1, \cdots, N\right\}$, onde $t_{j}$ é o tempo de chegada das ondas $\mathrm{P}$ em segundos em sensor localizado a $x_{j}$ kilômetros da fonte sísmica.

Representados sob a forma de segmentos de reta, os parâmetros dos grupos de fases podem ser definidos de forma a ressaltar suas diferentes características

$$
l_{k}=\left(\rho_{k}, \alpha_{k}, \bar{x}_{k}, \bar{t}_{k}, \boldsymbol{q}_{k}\right) \text {, }
$$

onde $l_{k}$ é o vetor que possui os seguintes parâmetros para o $k$-ésimo segmento de reta: $\rho_{k}$ e $\alpha_{k}$ consistem em suas coordenadas polares e os pontos $\left(\bar{x}_{k}, \bar{t}_{k}\right)$ são o centro de gravidade do segmento de rera gerado pelo grupo de fases contidos nos pontos de suporte $\boldsymbol{q}_{\boldsymbol{k}}$.

Definidos os parâmetros a partir da Equação 1, aplica-se o método Split-Merge and Fuzzy (SMF) para seleção e agrupamento dos vários segmentos de reta em grupos de onda Pg e Pn. Proposto por (Borges et al., 2000), o método Split-Merge and Fuzzy (SMF) foi inicialmente empregado na solução do problema da extração de segmentos de retas em imagens bidimensionais utilizadas em sistemas de navegação por robôs. O algoritmo SMF consiste basicamente na aplicação do método de classificação de agrupamento difuso por protótipo (prototype fuzzy clustering) em um senso de divisão e fusão (split and merge) dos segmentos de reta. O algoritmo de classificação por agrupamento difuso, diferentemente de outros métodos de classificação por agrupamento, não necessita de requisitos como compactação ou forma de agrupamento dos dados (Theodoridis et al., 2003). Seu objetivo concentra-se na minimização da seguinte função de custo quadrática (Davé et al., 1997)

$$
J(\beta, U ; Z)=\sum_{i=1}^{C} \sum_{j=1}^{N} u_{i j}^{m} d^{2}\left(x_{j}, t_{j}, \beta_{i}\right),
$$

onde $\beta_{i}$ representa o parâmetro do i-ésimo protótipo, $m \in(1, \infty)$ é o fator de ponderação difuso, $u_{i j}$ é o grau de membresia do j-ésimo ponto $\left(x_{j}, t_{j}\right)$ em relação ao protótipo $\beta_{i}$, e $d^{2}\left(x_{j}, t_{j}, \beta_{i}\right)$ é a função que determina a distância entre os pontos $\left(x_{i}, t_{i}\right)$ e o protótipo $\beta_{i}$. O número $C$ de agrupamentos, bem como o seu formato, devem ser estabelecidos a priori. O senso de divisão e fusão é empregado para contornar a determinação do número de agrupamentos para o problema da extração de segmentos de retas caracterizadas pelos parâmetros definidos na equação (1).

O método SMF emprega as etapas de divisão e fusão onde, na etapa de divisão, todos os segmentos de retas são gerados de forma iterativa, empregando-se o algoritmo de classificação por agupamento difuso por protótipo, obedecendo ao critério de validação $\delta_{k} \leq \delta_{\max }$, baseado na dispersão quadrática dos pontos $\left(x_{j}, t_{j}\right)$ em relação ao protótipo $\beta_{k}$

$$
\delta_{k}^{2}=\frac{1}{S_{k}} \sum_{j \in q_{k}} d^{2}\left(x_{j}, t_{j}, \beta_{k}\right),
$$

onde $s_{k}$ é o número de pontos de suporte. Ao decorrer da etapa de divisão, uma lista de segmentos de reta $L=\left\{\boldsymbol{l}_{\boldsymbol{k}} \mid k=1, \cdots, N_{L}\right\}$ é formada, onde $N_{L}$ é o número de segmentos gerados na etapa de divisão. A etapa de fusão corresponde na junção de um segmento $\boldsymbol{l}_{\boldsymbol{k}}$ com outros segmentos de reta. Caso o critério de validação seja verificado, a junção dos segmentos é considerada válida e $N_{L}$ é atualizado. Finaliza-se o algoritmo quando não for possível realizar mais nehuma fusão dentro do critério de validação. Em ambas as etapas, o limiar de validação $\delta_{\max }$ é um parâmetro livre e deve ser definido pelo usuário. Mais detalhes sobre o algoritmo SMF e a classificação de proptótipo por agrupamento difuso podem ser encontrados em (Borges et al., 2000), (Davé et al., 1997) e (Theodoridis et al., 2003).

De modo a localizar o ponto de mudança da onda direta $(\mathrm{Pg})$ para a refratada $(\mathrm{Pn})$, o algoritmo aplica regressão linear por mínimos quadrados ponderados nos segmentos de reta contidos em $L$.

No intuito de extrair seus parâmetros sob a forma $t=a x+b$, a equação é rearranjada da seguinte forma

$$
t=\hat{\varphi}(x)^{T} \theta,
$$

onde $\hat{\varphi}(x)^{T}=\left[\begin{array}{ll}x & 1\end{array}\right]$ e $\theta=\left[\begin{array}{ll}a & b\end{array}\right]$.

A regressão linear por mínimos quadrados ponderados é dada pela seguinte representação em lote (Theodoridis et al., 2003)

$$
\hat{\theta}=\left(\boldsymbol{\phi}^{T} \cdot W \cdot \boldsymbol{\phi}\right)^{-1} \cdot \boldsymbol{\phi}^{T} \cdot W \cdot T,
$$

onde $\boldsymbol{\phi}=\left[\phi\left(x_{1}\right) \ldots \phi\left(x_{n}\right)\right]^{T}$ e $T=\left[t_{1} \ldots t_{n}\right]$. Os pesos são representados pela matriz $W=\operatorname{diag}\left(w_{1} \ldots w_{n}\right)$ calculados pela função de pesos de Cauchy, representante da classe dos M-estimadores robustos (Huber,1981), dada por

$$
w_{n}=\frac{1}{1+\left(\frac{r_{n}}{c}\right)^{2}},
$$

onde $r_{n}=t_{n}-\hat{\varphi}\left(x_{n}\right)^{T} \theta$ são os resíduos do $n$-ésimo ponto avaliado e $c=2.3849$ é uma constante de ajuste do peso.

A classificação das primeiras chegadas como onda direta $(\mathrm{Pg})$ ou refratada na Moho $(\mathrm{Pn})$ é determinada pelo valor de $\hat{a}_{k}$ dentro da variável $\hat{\theta}_{k}$. Quando este valor atingir o limiar de vagarosidade $(\mathrm{s} / \mathrm{km})$ estabelecido pelo usuário, os segmentos de retas anteriores à $\boldsymbol{l}_{\boldsymbol{k}}$ são agrupados como onda direta $\boldsymbol{l}_{\boldsymbol{d}}$, e os segmentos posteriores como onda refratada $\boldsymbol{l}_{r}$. Os parâmetros de cada grupo $\theta_{d}$ e $\theta_{r}$ dos novos segmentos de reta são calculados por meio da regressão linear usando mínimos quadrados ponderados, mostrados nas equações (5) e (6).

Obtido os segmentos de reta das ondas direta $(\mathrm{Pg})$ e refratada $(\mathrm{Pn})$, a velocidade da onda $\mathrm{P}$ na crosta superior $v_{c}(\mathrm{~km} / \mathrm{s})$ é dada por (Stein et al, 2002)

$$
v_{c}=\frac{1}{a_{d}},
$$



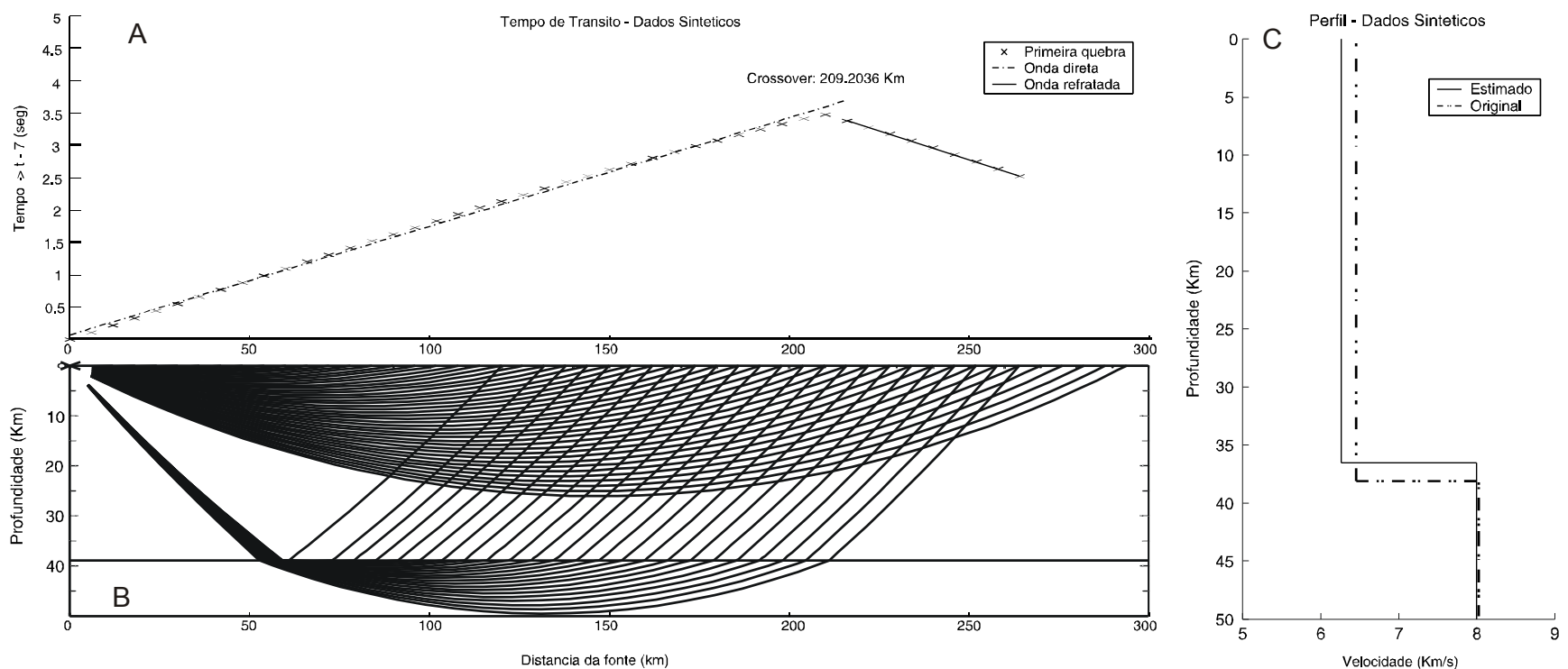

Figura 1-Resultados do algoritmo com dados sintéticos. Tempo de trânsito (A), traçado de propagação do raio (B) e modelo de uma dimensão reais e obtidos pelo método (C).

onde $a_{d}$ é o termo do parâmetro $\theta_{d}$ o qual corresponde a inclinação do segmento de reta da onda direta. De maneira análoga, a velocidade no manto $v_{m}$ é definida da seguinte maneira

$$
v_{m}=\frac{1}{a_{r}}
$$

onde $a_{r}$ é o termo do parâmetro $\theta_{r}$ o qual corresponde a inclinação do segmento de reta do grupo de fase da onda refratada.

O ponto espacial no qual o grupo das ondas refratradas aparecem como primeira quebra no sismograma (ponto de crossover) é determinado como

$$
x_{0}=\frac{b_{r}}{a_{d}-a_{r}},
$$

onde $b_{r}$ é o termo de $\theta_{r}$ que representa o offset do agrupamento de fases da onda refratada.

A espessura da crosta (em $\mathrm{km}$ ) é calculada utilizando a seguinte equação (Stein et al., 2002)

$$
h_{0}=\frac{x_{0}}{2\left(\frac{v_{m}+v_{c}}{v_{m}-v_{c}}\right)^{\frac{1}{2}}} \text {. }
$$

\section{Resultados}

A metodologia proposta foi aplicada em dados sintéticos e dados reais.

Tempos de trânsito sintéticos foram gerados com o pacote de traçado de raio SEIS88 (Cervený et al., 1977) para modelo crustal de uma camada com a descontinuidade de Moho a $38.7 \mathrm{~km}$ de profundidade, velocidade da onda $P$ de $6.2-6.7 \mathrm{~km} / \mathrm{s}$ do topo para a base da crosta e $P$ do manto de $8.1 \mathrm{~km} / \mathrm{s}$ (Fig.1). Para o espaço de dados considerado, utilizou-se o fator de dispersão $\delta_{\max }^{2}=7$. Os tempos de trânsito sintéticos, juntamente com o resultado do algoritmo proposto, estão mostrados na Tabela 1 e na Figura 1A. O traçado de raio possui efeito ilustrativo pode ser visualizado na Figura $1 \mathrm{~B}$.

Tabela 1 -Característica sísmica do modelo sintético e do modelo obtido pelo algoritmo

\begin{tabular}{|l|c|c|c|}
\hline \multirow{2}{*}{$\begin{array}{c}\text { Base de } \\
\text { Dados }\end{array}$} & \multicolumn{2}{|c|}{ Velocidade $\mathbf{( k m / s )}$} & \multirow{2}{*}{ Espessura (km) } \\
\cline { 2 - 3 } & $v_{c}$ & $v_{m}$ & \\
\hline Sintéticos & 6.45 & 8.00 & 38.1 \\
\hline Algoritmo & 6.25 & 8.01 & 36.7 \\
\hline
\end{tabular}

Os resultados obtidos com o algoritmo apresentam $v_{c} \mathrm{e}$ espessura da crosta menor que a do modelo e $v_{m}$ compatível com o valor original. Como mostrado no traçado de raio da Figura $1 \mathrm{~B}$, a onda $\mathrm{Pg}$ atravessa apenas a parte rasa da crosta, produzindo velocidade representativa da crosta superior, conseqüentemente menor que a velocidade média da crosta. A crosta continental normalmente apresenta aumento na velocidade das ondas sísmicas a aproximadamente 25$30 \mathrm{~km}$ de profundidade, marcando o início da crosta inferior. O aumento na velocidade é resultado de mudança de composição (félsica-máfica) e/ou aumento do grau metamórfico das rochas. As fases refratadas nesta descontinuidade não aparecem como primeiras chegadas, são normalmente fases secundárias nos sismogramas (Giese et al.,1976a).

A crosta inferior apresenta velocidade maior que a crosta superior, de forma que a velocidade média da crosta aumenta quando a crosta inferior é considerada. Para velocidade crustal menor que a real a espessura obtida para a crosta será também menor que a real, gerando modelo crustal de espessura mínima. 

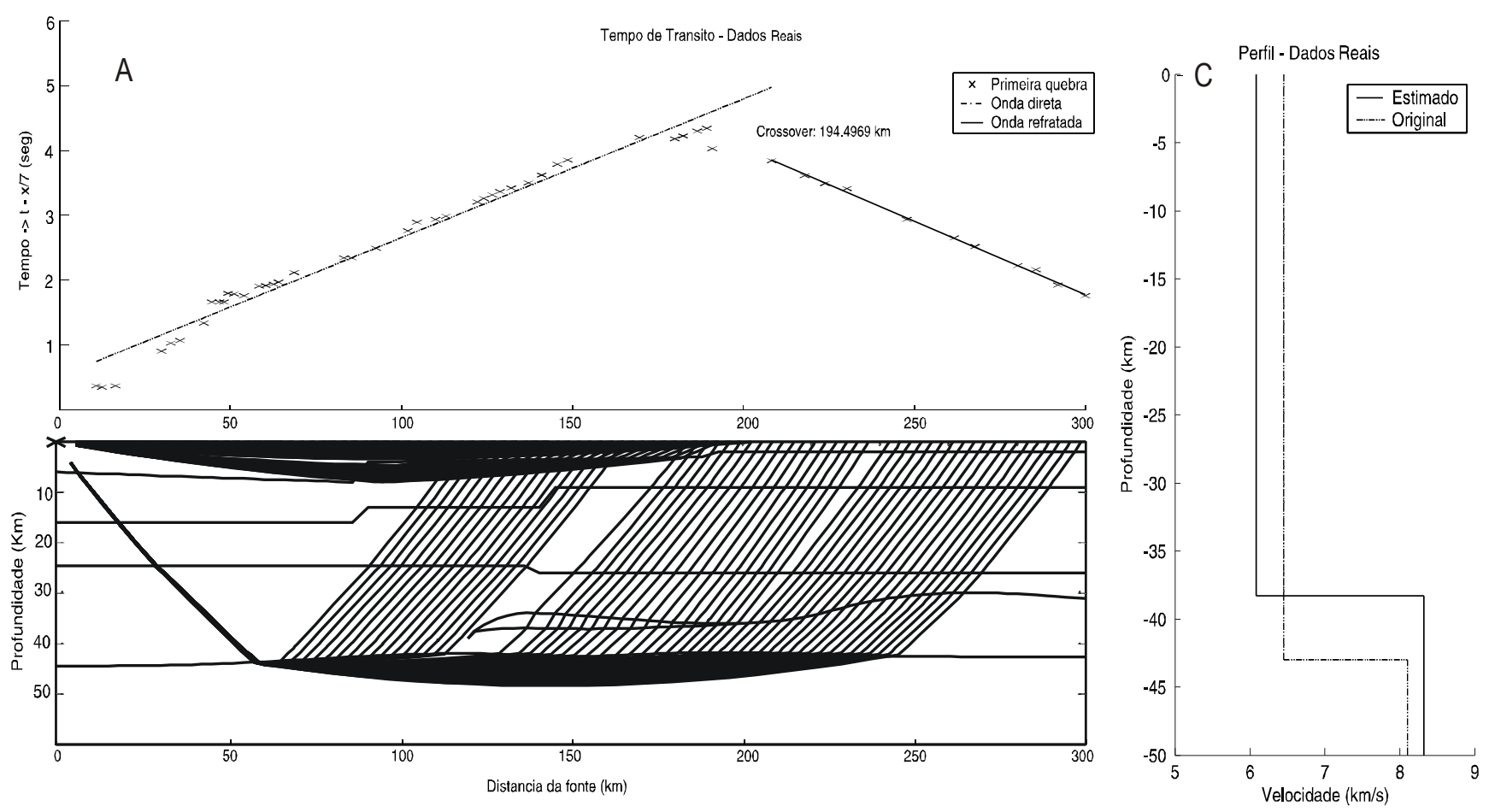

Figura 2 -Resultados do algoritmo com dados reais utilizados em estudos de refração sísmica profunda na região de Cavalvante. Tempo de trânsito (A), traçado de propagação do raio (B) e modelo 1D obtidos pelo método (C).

A Figura 2A mostra a leitura das primeiras chegadas do tiro 1 da linha de Cavalcante e o resultado da classificação realizada pelo algoritmo SMF.O algoritmo foi implementado com fator de dispersão $\delta_{\max }^{2}=15$. A Figura $2 \mathrm{C}$ e a Tabela 2 apresentam os resultados obtidos pelo algoritmo comparados com aqueles obtidos pela modelagem direta 1D (Soares, 2005). De forma geral comprovam os resultados obtidos pelos dados sintéticos. A Vm ficou bem determinada, o que é percebido pelo ajuste perfeito das fases refratadas (Figura 2A). A diferença na profundidade da Moho entre aquela obtida pela modelagem direta 1D e aquela obtida pelo algoritmo reflete o erro na determinação da velocidade média da crosta $v_{c}$. No caso de Cavalcante as primeiras quebras apresentam pelo menos dois alinhamentos bem definidos antecedendo o alinhamento de fases da Moho. O algoritmo aproximou essas fases para um único alinhamento, falseando para menos a velocidade média da crosta superior e, conseqüentemente, aumentando o erro na determinação da profundidade da Moho.

Tabela 2 -Modelo $1 D$ do tiro 1 da linha de refração sísmica profunda de Cavalcante e modelo com espessura obtido pelo algoritmo.

\begin{tabular}{|l|c|c|c|}
\hline \multirow{2}{*}{$\begin{array}{c}\text { Base de } \\
\text { Dados }\end{array}$} & \multicolumn{2}{|c|}{ Velocidade $\mathbf{( k m / s )}$} & \multirow{2}{*}{ Espessura (km) } \\
\cline { 2 - 3 } & $v_{c}$ & $v_{m}$ & \\
\hline Cavalcante & 6.42 & 8.28 & 43 \\
\hline Algoritmo & 6.08 & 8.32 & 38.3 \\
\hline
\end{tabular}

\section{Discussão e Conclusões}

Os métodos Split-Merge and Fuzzy (SMF) e mínimos quadrados ponderados foram aplicados com sucesso na identificação automática dos grupos de fases e determinação automática do alinhamento de fases correspondentes à onda direta $(\mathrm{Pg})$ e refratada na Moho (Pn) de um levantamento de refração sísmica profunda.

O erro na determinação da profundidade da Moho está diretamente ligado ao erro na determinação da velocidade média da crosta. O algoritmo aproxima a velocidade média da crosta pelo alinhamento das primeiras chegadas anteriores à chegada da refração na Moho. Essas fases $(\mathrm{Pg})$ trazem informação sobre a velocidade da crosta superior, gerando modelo crustal com a Moho sempre mais rasa do que a real. $O$ algoritmo gera modelo com a profundidade mínima da Moho. O modelo de profundidade mínima será usado na determinação da janela de busca para determinação automática da reflexão da Moho (PmP), próximo passo na construção do algoritmo.

A velocidade do manto litosférico $\left(v_{m}\right)$ é determinada com precisão. Aplicado em tiros diretos e reversos de um levantamento 0 algoritmo pode detectar possíveis inclinações na descontinuidade de Moho. Em comparação com valores previamente estabelecidos, o algoritmo apresentou desvios em $v_{m}$ da ordem de 0.01 $\mathrm{km} / \mathrm{s}$ para os dados sintéticos e $0.04 \mathrm{~km} / \mathrm{s}$ para os dados utilizados em Cavalcante.

Este trabalho propôs a utilização de um método automático para obtenção de estimativas iniciais acerca da estrutura crustal em uma linha de refração sísmica. A partir do tempo de trânsito das primeiras chegadas em levantamentos de refração sísmica profunda, o algoritmo proposto foi capaz de classificar os grupos de ondas 
compressionais diretas $(\mathrm{Pg})$ e refratadas pela Moho $(\mathrm{Pn})$. Apesar da considerável incerteza associada aos valores calculados da velocidade média na crosta, desencadeando um erro considerável na determinação da espessura da crosta, este trabalho consiste em uma etapa intermediária na pesquisa de um paradigma automático para geração de um modelo 1D da crosta continental. A automatização das tarefas de detectar e classificar o grupo de ondas presentes nas primeiras chegadas proporcionam a diminuição no tempo gasto na análise manual, dado o grande volume de dados utilizados para estabelecer um modelo crustal inicial.

A próxima etapa consiste estabelecer uma metodologia para o cálculo da velocidade média da crosta $v_{c}$ a partir da determinação do tempo de trânsito das fases secundárias PmP. De acordo com (Giese et al., 1976b), a determinação de $v_{c}$ a partir da leitura das fases Pmp, fornecem é mais precisa devido a sua imunidade às interferências causadas pela refração na Moho.

\section{Referências}

BERROCAL, J., MARANGONI, Y., CÔGO DE SÁ, N., FUCK, R.A., SOARES, J.E.P., DANTAS, E., PEROSI, F., \& FERNANDES, C.-2004- Deep seismic refraction and gravity crustal model and tectonic deformation in Tocantins Province, Central Brazil. Tectonophysics, 388, 187-199.

BORGES, G.A. \& ALDON, M.-2000- A Split-and-Merge segmentation algorithm for line extractions in 2-D range images, In Proc of $15^{\text {th }}$ International Conference on Pattern Recognition.

CHRISTENSEN, N.I \& MOONEY, W.D.-1995- Seismic velocity structure composition of the continental crust: $A$ global view, Journal of Geophysical Research, 100(B7), pp. 9761-9788.

DAVÉ, R.N. \& Krishnapuram, R.-1997- Robust Clustering Methods: A Unified View, IEEE Transactions on Fuzzy Systems, 5(7), pp. 270-293.
ČERVENÝ, V. Molotkov, I.A. and PŠENČíK-1977- Ray Methods in Seismology, University of Karlova Press, Prague.

FUCK, R.A., BERROCAL, J., ASSUMPÇÃO, M. e outros2006- Estudos geofísicos e tectônicos na Província Borborema. Projeto pesquisa financiado pelo CNPq, rubrica Instituto do Milênio.

GIESE, P-1976a- Problems and Tasks of Data Generalization. In Explosion Seismology in Central Europe (P. Giese, C. Prodehl \& A. Stein, ed.). Deutsche Geophysikalische Gesellcshaft. Springer-Verlag, New York, USA.

GIESE, P-1976b- Depth Calculation. In Explosion Seismology in Central Europe (P. Giese, C. Prodehl \& A. Stein, ed.). Deutsche Geophysikalische Gesellcshaft. Springer-Verlag, New York, USA.

HUBER, P.J.-1981- Robust Statistics, John Wiley \& Sons, New York, USA.

MELO, F.X. \& BORGES, G.A. -2005- P-Wave Onset Time Determination Based On Background Noise Autoregressive Model Tracking with Kalman Filtering, In: IASPEI General Assembly, Santiago, Chile.

MELO, S.S.V.-2005- Razão de Poisson da crosta superior da região de Porangatu, Goiás, Província Tocantins: um estudo por refração sísmica. Universidade de Brasília, Instituto de Geociências, Brasília. Tese de mestrado 203

SOARES, J.E.P-2005- Estudos de refração sísmica profunda, função do receptor e gravimetria na Província Tocantins, Brasil central. Universidade de Brasília, Instituto de Geociências, Brasília. Tese de doutorado 73

STEIN, S. \& WYSESSION, M.-2002- An Introduction to Seismology, Earthquakes, and Earth Structure. Blackwell Publisinhg, Oxford, UK.

THEODORIDIS, S. \& KOUTROUMBAS, K.-2003- Pattern Recognition, $2^{\text {nd }}$ ed, Elsevier, San Diego, USA. 